\section{Band 18, Heft 3, Juni 2011}

\section{Editorial}

120 Verantwortung - die verkannte Ressource Walach, H. (Frankfurt/O.)

\section{Originalarbeiten}

122 Medizinische Schröpftherapie bei 30 Patienten mit Fibromyalgie: Eine Fallserienbeobachtung

Cao, H.; Hu, H.; Liu, J. (Beijing)

127 Die Bedeutung einer kunsttherapeutischen Intervention für onkologische Patienten aus Sicht der Teilnehmer, der Kursleitung und der Supervisorin Geue, K.; Buttstädt, M.; Singer, S.; Kleinert, E.; Richter, R.; Götze, H.; Böhler, U.; Becker, C.; Brähler, E. (Leipzig)

\section{Übersichtsarbeiten}

134 Topische Kräutermedizin in der Behandlung von diabetischer peripherer Neuropathie: Eine systematische Übersichtsarbeit über randomisierte kontrollierte Studien

Chen, W.; Luo, Y.F. (Beijing); Liu, J.P. (Beijing, Troms $\varnothing$ )

146 Energieheilung bei Krebs: Ein kritischer Review Agdal, R.; Hjelmborg, J.v.B.; Johannessen, H. (Odense)

\section{Brief an die Herausgeber}

155 Unsere systematischen Reviews sind wichtig und relevant - Antwort auf Vickers

Lee, M.S.(Daejeon); Ernst, E. (Exeter)

\section{Journal Club}

156 Ein wenig Sand in die Augen gestreut

157 Mind-Body bei Fibromyalgie

159 Biodiesel bei Karies

160 Tausendsassa Grüner Tee: Mehr größere Studien nötig
Vol. 18, Issue 3, June 2011

\section{Editorial}

120 Responsibility - the Underestimated Resource Walach, H. (Frankfurt/O.)

\section{Original Articles}

122 Medicinal Cupping Therapy in 30 Patients with Fibromyalgia: A Case Series Observation

Cao, H.; Hu, H.; Liu, J. (Beijing)

127 The Impact of an Art Therapy Program for Cancer Patients - an Analysis from Different Points of View Geue, K.; Buttstädt, M.; Singer, S.; Kleinert, E.; Richter, R.; Götze, H.; Böhler, U.; Becker, C.; Brähler, E. (Leipzig)

\section{Review Articles}

134 Topical Herbal Medicine for Treatment of Diabetic Peripheral Neuropathy: A Systematic Review of Randomized Controlled Trials Chen, W.; Luo, Y.F. (Beijing); Liu, J.P. (Beijing, Troms $\varnothing$ )

146 Energy Healing for Cancer: A Critical Review Agdal, R.; Hjelmborg, J.v.B.; Johannessen, H. (Odense)

Letter to the Editors

155 Our Systematic Reviews Are Important and Relevant - Reply to Vickers Lee, M.S.(Daejeon); Ernst, E. (Exeter)

156 Journal Club

156 To Pull Some Wool over Our Eyes

157 Mind-Body for Fibromyalgia

159 Biodiesel for Caries

160 Jack of All Trades Green Tea: More Substancial Studies Are Needed

\section{KARGER}

Fax +497614520714

Information@Karger.de

www.karger.com
(C) 2011 S. Karger GmbH, Freiburg 


\section{Komplementärmedizin}

Wissenschaft • Praxis • Perspektiven

\section{Band 18, Heft 3, Juni 2011}

Abstract Service

162 Latest Publications You Should Not Miss

164 Buchbesprechung

165 Fort- und Weiterbildung

166 News / Ticker

167 Tagungen und Kongresse

121 Impressum

U3 Hinweise für Autoren (3. Umschlagseite)
Vol. 18, Issue 3, June 2011

Abstract Service

162 Latest Publications You Should Not Miss

$\mathbf{1 6 4}$ Book Review
$\mathbf{1 6 5}$ Education
$\mathbf{1 6 6}$ News / Ticker
$\mathbf{1 6 7}$ Meetings and Conferences
$\mathbf{1 2 1}$ Imprint
C3 Guidelines for Authors (Inside back cover) 